\title{
Efectos económicos de la pandemia del Covid-19 en el consumo de hortalizas en los hogares en la ciudad de Cúcuta, Colombia
}

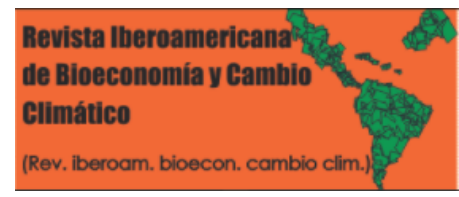

\author{
Economic effects of the Covid-19 pandemic on household \\ vegetable consumption in the city of Cúcuta, Colombia
}

\author{
Núñez R., J.J; Arámbula G., C.I; González V., M.S; Carvajal R., J.C; \\ Editor Académico Prof. M Sc. Edgar Antonio Marineros-Orantes
}

(iD J.J Núñez R.

jo.nunez@mail.udes.edu.co

Universidad de Santander, Colombia

iD C.I Arámbula G.

arambula@mail.udes.edu.co

Universidad de Santander, Colombia

iD M.S González V.

ma.gonzalez@mail.udes.edu.co

Universidad de Santander, Colombia

iD J.C Carvajal R.

jul.carvajal@mail.udes.edu.co

Universidad de Santander, Colombia

Editor Académico Prof. M Sc. Edgar Antonio

Marineros-Orantes

Universidad de El Salvador, El Salvador

Revista Iberoamericana de Bioeconomía y Cambio Climático

Universidad Nacional Autónoma de Nicaragua, León, Nicaragua ISSN-e: 2410-7980

Periodicidad: Semestral

vol. 7 , núm. 14, 2021

czuniga@ct.unanleon.edu.ni

Recepción: 08 Noviembre 2021

Aprobación: 11 Diciembre 2021

URL: http://portal.amelica.org/ameli/

jatsRepo/394/3941761014/index.html

DOI: https://doi.org/10.5377/ribcc.v7i14.12946

Autor de correspondencia: jo.nunez@mail.udes.edu.co
Resumen: La pandemia del Covid-19 ha afectado el sistema agroalimentario del país, en sus cadenas productivas desde la oferta, comercialización y consumo de alimentos en los hogares. Dentro este marco ontológico se desarrolla el proyecto de investigación "Impactos de la pandemia del Covid-19 en la seguridad agroalimentaria del departamento Norte de Santander 2020-2021", obteniendo resultados parciales en los efectos de la pandemia en el consumo de hortalizas en los hogares de la ciudad de Cúcuta, capital del departamento. Se consultaron bases de datos de CENABASTOS y MINAGRICULTURA (2019-2020) y a jefes de familias mediante una encuesta online a 323 hogares de los miembros de la comunidad de la Universidad de Santander (UDES) en el período enero-julio de 2021 sobre las variaciones en la cantidad, calidad, precio y frecuencia del consumo de las principales hortalizas que se producen en el departamento (papa, tomate, pimentón, cebolla y ajo). Los resultados indican que al comparar los precios de los años 2019 (antes de la pandemia) y 2021 (tercer pico de la pandemia) se evidencian incrementos de los precios de la papa en un $97,90 \%$, tomate un $2,39 \%$, cebolla blanca cabezona en un $105,02 \%$, ajo en un $23,79 \%$ y zanahoria en un $2,53 \%$. En las encuestas la percepción de los jefes de hogar señala que la cantidad de hortalizas consumidas han sido afectadas en un $42,48 \%$, la calidad en un $37,08 \%$, los precios en el $49,59 \%$ y la frecuencia en un $33,08 \%$.

Palabras clave: Sistema agroalimentario, Covid-19, Seguridad alimentaria, Oferta, Demanda.

\begin{abstract}
The Covid-19 pandemic has affected the agrifood system of the country, in its productive chains from the supply, marketing and consumption of food in households. Within this ontological framework, the research project "Impacts of the Covid-19 pandemic in the agrifood security of the department of Norte de Santander 2020-2021" is being developed, obtaining partial results on the effects of the pandemic in the consumption of vegetables in the homes of the city of Cúcuta, capital of the department. Databases of CENABASTOS and MINIAGRICULRURA 2019, 2020) and heads of families were consulted through an online survey of 323 households of members of the community of the University of Santander (UDES) in the period January-July 2021 on
\end{abstract}


variations in the quantity, quality, price and frequency of consumption of the main vegetables produced in the department (potato, tomato, paprika, onion and garlic). Results indicate that when comparing the prices of the years 2019 (before the pandemic) and 2021 (third peak of the pandemic), price increases of potato by $97.90 \%$, tomato by $2.39 \%$, white onion by $105.02 \%$, garlic by $23.79 \%$ and carrot by $2.53 \%$ are evidenced. In the surveys, the perception of the heads of household indicates that the quantity of vegetables consumed has been affected by $42.48 \%$, quality by $37.08 \%$, prices by $49.59 \%$ and frequency by $33.08 \%$.

Keywords: Agri-food system, Covid-19, Food security, Supply, Demand.

\section{INTRODUCCIÓN}

La Organización Mundial de la Salud (OMS) el 11 de marzo 2020 declara al coronavirus (Corona virus COVID-19) como una pandemia global. A Colombia el Covid - 19 llega en el mes marzo de 2020 y el día 24 el gobierno declara la cuarentena nacional (Tikitakas, 2020). Esta medida de protección de la población paraliza la vida cotidiana de la sociedad en todos sus ámbitos, afectando con mayor fuerza los sectores económicos y sociales en cuanto a la producción de bienes y servicios, hecho que perturba el empleo y la generación de ingresos de las familias.

Numerosos organismos nacionales e internacionales (Organización de las Naciones Unidas para la Agricultura y la Alimentación (FAO). (2020); el Programa Mundial de Alimentos (PMA). (2020); Organización Mundial del Comercio (OMC). (2020) han venido alertando sobre las secuelas del Covid-19, junto al cambio climático, al agudizar la crisis de la seguridad alimentaria en países pobres, o en desarrollo, con el riesgo de hambrunas en los sectores vulnerables de la población.

La seguridad alimentaria nutricional "es un estado en el cual todas las personas gozan, en forma oportuna y permanente, de acceso físico, económico y social a los alimentos que necesitan, en cantidad y calidad, para su adecuado consumo y utilización biológica, garantizándoles un estado de bienestar general que coadyuve al logro de su desarrollo" (Instituto de Nutrición para Centroamérica y Panamá (INCAP) citado por PESA, 2011), comportando la disponibilidad, acceso y uso de los alimentos (PMA, 2008).

La vulnerabilidad multisectorial del departamento Norte de Santander antes de la pandemia era significativa al compararla con las demás entidades del país. Datos oficiales evidencian la difícil situación económica y social de la entidad al tener un 14,6\% de desempleo (Departamento Administrativo Nacional de Estadística, DANE, 2019), 73,3\% de informalidad en la ciudad de Cúcuta (Diario La Opinión, 11-10-2019), ocupar el puesto 12 en el índice departamental de competitividad (Diario La Opinión, 26-11-2019), 31,5\% del índice de pobreza multidimensional (Programa de Naciones Unidas para el Desarrollo (PNUD. (2019) y contener una población migrante venezolana de 183.215 desplazados, para el mes de diciembre de 2019 (Diario Frontera, 24-12-2019), que hacía más compleja y precaria la realidad de Norte de Santander.

Asimismo, en el ámbito de la disponibilidad de alimentos el sector agropecuario del departamento está conformado predominantemente por una agricultura familiar de subsistencia (Núñez, 2018). Las frágiles condiciones de las vías de comunicación desde las fincas hasta los mercados, el bajo nivel de tecnificación agropecuaria, las deficientes condiciones de las infraestructuras y servicios en las zonas rurales y los deficientes

Notas DE AUTOR

jo.nunez@mail.udes.edu.co 
canales de comercialización (Núñez y Carvajal, 2014) otorgan, además, una significativa vulnerabilidad en la oferta de alimentos a las principales ciudades del departamento. Otro de los aspectos a considerar se refiere a que la oferta de alimentos producidos en el departamento no es suficiente para atender la demanda local, por lo que se requiere complementar con productos traídos de otros departamentos del país por vía aérea y terrestre, dificultando el acceso a los alimentos por las restricciones propias de la pandemia.

El objetivo de este artículo es analizar los efectos económicos de la pandemia del Covid-19 en el consumo de las principales hortalizas en los hogares de la ciudad de Cúcuta con el fin de establecer los impactos en la cantidad, calidad, frecuencia y precios durante el periodo 2019-2021.

\section{Materiales y MÉtodos}

La investigación se desarrolló en el departamento Norte de Santander, ubicado en la parte nororiental de Colombia; con una extensión es de $21.648 \mathrm{~km}^{2}$, representando el $1.89 \%$ del territorio nacional y con una población proyectada por la Departamento Administrativo Nacional de Estadistica (DANE) para el 2016 de 1.367.708 habitantes (2,8\% de participación nacional) (Gobernación del departamento Norte de Santander, 2016). En la producción de hortalizas en el país, antes de la pandemia, indicaba una tasa positiva en el área cosechada del 4\% (Ministerio de Agricultura (MINAGRICULTURA). (2019).

La estimación de los impactos en los parámetros económicos de la cadena productiva de valor de hortalizas en el departamento Norte de Santander se abordó desde la metodología cuantitativa de nivel descriptivo al basarse en la medición de variables, analizar y comprobar información y datos (Hernández et al., 2014). Se realizaron consultas en bases de datos estadísticos, período 2019-2021, de 6 organizaciones (DANE, SIPSA, AGRONET-MINAGRICULTURA, CENABASTOS, CORABASTOS, Secretaria de Agricultura y Desarrollo Rural) sobre producción, comercialización y precios a nivel de mercados mayoristas de Cúcuta y Bogotá. Asimismo, se aplicó una encuesta on line, mediante la herramienta Lime Survey (González, 2011), a una muestra de 323 miembros de la comunidad universitaria de los estratos socioeconómicos 1 al 6, compuesta por el 58,3\% de mujeres y 41,7\% de hombres entre las edades de 18 a 60 años de edad. Los componentes de la encuesta se corresponden con las categorías de cantidad, calidad, precios y frecuencia de consumo de hortalizas durante la pandemia. Se utilizó la escala de intensidad de respuestas: Ha afectado mucho (A1), ha afectado medianamente (A2), ha afectado poco (A3), no ha afectado ni beneficiado (A4), ha beneficiado poco (A5), ha beneficiado medianamente (A6) y ha beneficiado mucho (A7).

\section{Resultados y Discusión}

\section{Tendencias en la producción de hortalizas antes y durante la pandemia}

En las Tablas 1 y 2 se muestra el comportamiento en la producción de hortalizas a nivel nacional y en el departamento Norte de Santander

TABLA 1

Indicadores de producción de hortalizas a nivel nacional

\begin{tabular}{lllll}
\hline $\begin{array}{l}\text { Variable } \\
\text { Area cosechada } \\
\text { (ha) }\end{array}$ & 2016 & 2017 & 2018 & $2019^{*}$ \\
$\begin{array}{l}\text { Producción (ton) } \\
\text { Rendimiento }\end{array}$ & 2.095 .615 & 83.712 & 85.386 & 87.094 \\
(ton/ha) & 24,63 & 25,18 & 25,18 & 25,18 \\
\hline
\end{tabular}


Fuente: MINAGRICULTURA (2019). * Estimado DCAF

TABLA 2.

Indicadores de producción de hortalizas en el departamento Norte de Santander

\begin{tabular}{|c|c|c|c|c|}
\hline Variable & 2016 & 2017 & $2018^{*}$ & $2019^{*}$ \\
\hline $\begin{array}{l}\text { Area cosechada } \\
\text { (ha) }\end{array}$ & 7.646 & 6.562 & 6.693 & 6.827 \\
\hline Producción (ton) & 177.315 & 153.952 & 15 & 160.172 \\
\hline $\begin{array}{l}\text { Rendimiento } \\
\text { (ton } / \text { ha) }\end{array}$ & 23,19 & 23,46 & 23,46 & 23,46 \\
\hline
\end{tabular}

Fuente: Elaboración propia con datos de MINAGRICULTURA (2019). ${ }^{*}$ Estimado DCAF

En general, la producción de hortalizas se ubica dentro de la economía campesina, son pequeños productores con predios menores a 3 has. de superficie, producen productos frescos para un mercado interno (MINAGRICULTURA, 2019). Esta fuente afirma que el consumidor colombiano es poco exigente en calidad e inocuidad de los alimentos y que la cadena productiva de las hortalizas genera unos 220.000 empleos, de los cuales 70.000 son directos y 150.000 son empleos indirectos.

\section{Efectos económicos de la pandemia del Covid-19 en la comercialización de hortalizas}

El comportamiento de los precios de las hortalizas en estudio en los últimos 4 años (2018-2021, 01 de marzo) en las principales centrales de abastos del país (Bogotá, Armenia, Arauca y Cúcuta), reportadas por AGRONET (2021), se presentan en la Tabla 3 y Figura 1.

TABLA 3

Comportamiento de los precios de las hortalizas, periodo 2018-2021(01)

\begin{tabular}{|c|c|c|c|c|c|c|}
\hline & Precios & Precio & Precios & Precios & Precios & Variación \\
\hline & $\$ 1 \mathrm{~kg}$ & $\$ / \mathrm{kg}$ & $\$ 1 \mathrm{~kg}$ & $\$ 1 \mathrm{~kg}$ & $\$ / \mathrm{kg}$ & $2020-2021$ \\
\hline Rubros & 2018 & 2019 & 2020 & $2021^{*}$ & Promedio & (\%) \\
\hline Papa & 759 & 622 & 439 & 1231 & 762,8 & 97,90 \\
\hline Tomate & 2142 & 2767 & 2833 & 2833 & 2643,8 & 2,39 \\
\hline $\mathrm{Ceb}$ & 13 & 856 & 11 & 1755 & 12 & 02 \\
\hline Pimentón & 3602 & 4653 & 3668 & 3979 & 3975,5 & -1 \\
\hline Ajo & 6865 & 8875 & 10025 & 10986 & 9187.8 & 23.79 \\
\hline Zanahoria & 2142 & 2763 & 2833 & 2833 & 2642,8 & 2,53 \\
\hline
\end{tabular}

Fuente: Elaboración propia con datos de AGRONET-MINAGRICULTURA (2021). ${ }^{*} 01$ marzo 2021 


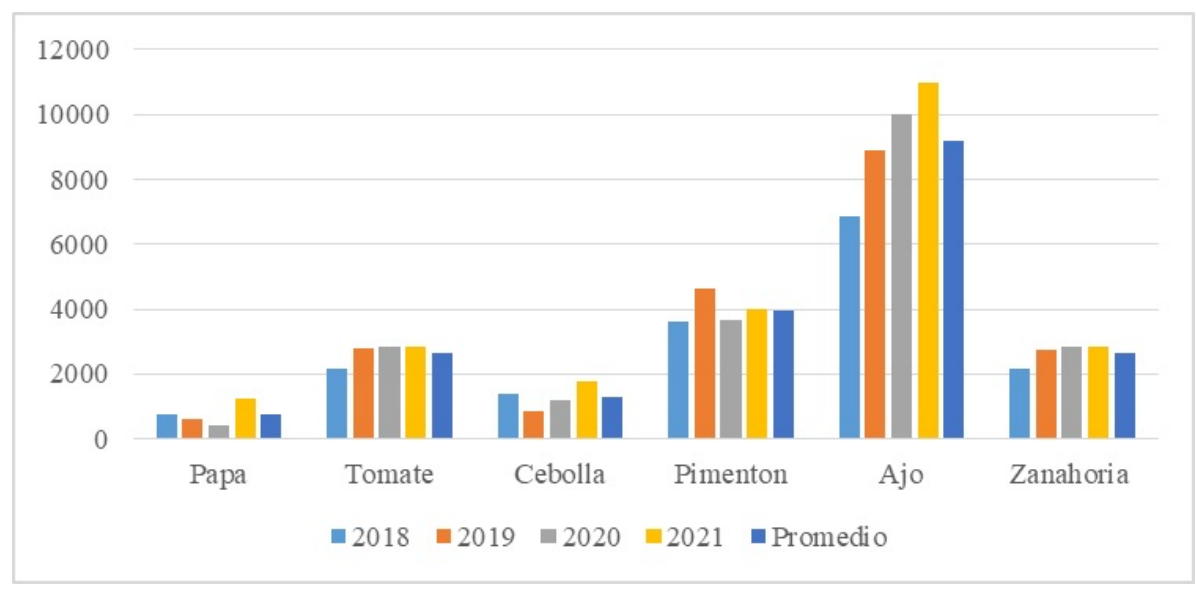

FIGURA 1.

Comportamiento de los precios de las hortalizas, periodo 2018-2021

Fuente: Elaboración propia con datos de AGRONET- MINAGRICULTURA (2021)

En el período observado se evidencia que en casi todas las hortalizas en estudio los precios de los años 2020 y 2021 (pandemia del Covid-19) fueron superiores a los años precedentes. En el caso de la papa el precio por kilogramo en los mercados mayoristas pasó de $622 \$ / \mathrm{kg}$ en 2019 a $1231 \$ / \mathrm{kg}$ en el primer trimestre del 2021, lo que significa un $97,91 \%$ de incremento y un $22,64 \%$ superior con respecto al promedio de los 4 años analizados. En el año 2020 se observó una disminución del precio del $57,55 \%$ con respecto a la media general.

En el período observado se evidencia que en casi todas las hortalizas en estudio los precios de los años 2020 y 2021 (pandemia del Covid-19) fueron superiores a los años precedentes. En el caso de la papa el precio por kilogramo en los mercados mayoristas pasó de $622 \$ / \mathrm{kg}$ en 2019 a $1231 \$ / \mathrm{kg}$ en el primer trimestre del 2021 , lo que significa un $97,91 \%$ de incremento y un $22,64 \%$ superior con respecto al promedio de los 4 años analizados. En el año 2020 se observó una disminución del precio del $57,55 \%$ con respecto a la media general.

En el precio del tomate se evidencia incremento del precio del 2,39\% entre los años 2019 y 2021 (marzo) y una disminución del 6,68\% en los años 2020 y 2021 con respecto al promedio de los 4 años de registros; la cebolla blanca de bulbo (cabezona) experimentó un incremento de 105,02\% al pasar el precio del kilogramo de 856 \& en 2019 a 1755 \$ en el primer trimestre del 2021 y superior en $35,86 \%$ con respecto a la media del periodo analizado. El pimentón fue el único rubro que no evidencia incrementos de precios durante el período de la pandemia 2020 y 2021. Su precio en el año 2019 se ubicó en $4.653 \$ / \mathrm{kg}$. y durante el primer trimestre de 2021 fue de $3.979 \$ / \mathrm{kg}$., es decir disminuyó en un 14,49\%. El precio del ajo en 2019 en las centrales mayoristas registró un promedio de $8.875 \$ / \mathrm{kg}$., en el año 2020 de $10.025 \$ / \mathrm{kg}$., y en marzo de 2021 de $10.986 \$ / \mathrm{kg}$, observándose un incremento del $23,79 \%$ con respecto al precio del 2021 y un $8,98 \%$ con relación a la media del periodo evaluado. Y, por último, con respecto a la zanahoria en el año 2019 tuvo un precio promedio de $2.763 \$ / \mathrm{kg}$. y subió durante los años 2020 y 2021 a $2.833 \$ / \mathrm{kg}$, lo que significa que aumentó un 2,53\% en el lapso en referencia.

A mediados del 2021 los precios de las hortalizas se han incrementado hasta en un 300\%, con respecto al mes de abril, debido al paro nacional que causó desabastecimiento en los principales mercados por el bloqueo de las vías en las zonas productoras y en las entradas a los sitios de consumo, hecho que sumado a los efectos de la pandemia del Covid-19 están generando grandes pérdidas económicas en la cadena productiva de hortalizas, especialmente en los eslabones de los productores hortícolas y comercializadores, y alzas importantes de los precios a nivel de los consumidores.

Los resultados obtenidos en el análisis del comportamiento de los precios de las hortalizas en los mercados mayoristas de Colombia coinciden con estudios realizados en Paraguay, Argentina y Colombia al observar incrementos en los precios de las hortalizas por efectos de las medidas restrictivas implementadas para frenar el contagio por el Covid-19, y en particular por el paro nacional en Colombia. 
El Centro de Análisis y Difusión de la Economía Paraguaya (CADEP). (2020) determinó efectos negativos de la pandemia en la oferta de hortalizas por escasez y aumento de precios en las semillas y alta incertidumbre por riesgos en comercialización de los productos, que implicaron contracciones en la demanda y una oferta limitada para los consumidores. Entre los factores que influyen en las variaciones de la oferta, demanda y precios de las hortalizas se destacan la alta sensibilidad shocks por eventos extremos, como la pandemia; y los efectos del confinamiento en la logística de la distribución y en las variaciones de los precios por temores de los consumidores al desabastecimiento (Centro Latinoamericano para el Desarrollo Rural, RIMISP, 2021), ocasionados por el cierre de ferias, mercados y supermercados Gutiérrez et al., (2021). En Colombia, la producción y comercialización de la papa y hortalizas se vieron afectadas por las variaciones climáticas, los bloqueos de vías por el paro nacional y las restricciones de la pandemia, derivando en una caída en la demanda y precios a nivel de los agricultores que perjudicaron a los agricultores por una sobre oferta productos (Ordinola y Devaux, 2021) y aumentos de los precios para los consumidores por escasez en la oferta disponible.

Con respecto a los aumentos puntuales en los precios de las hortalizas, en Paraguay, Sanabria-Velásquez et al., (2020) estimaron aumentos en los precios en hortalizas y legumbres del $15.79 \%$ durante los primeros 15 días de la cuarentena. Las hortalizas con mayor incremento fueron la cebolla cabezona con $31 \%$, zanahoria con $30.2 \%$, repollo con $30.7 \%$ y la papa con $23.5 \%$; en Argentina, aumentos del $77 \%$ de los precios de raíces y tubérculos y $58 \%$ en legumbres (Universidad Nacional de Rosario, 2021) y; en Colombia incrementos en los precios de la papa y la cebolla de un 16\% (RIMISP, 2021), valores inferiores a los estimados en esta investigación.

\section{Percepciones sobre los efectos de la pandemia del Covid-19 en la cantidad de hortalizas consumidas en los hogares}

Los resultados de la encuesta indican que la cantidad de hortalizas consumidas en los hogares durante la pandemia del Covid-19 ha tenido impactos negativos, sumar en el lado de las afectaciones un 42,48\%, sin efectos el 37,50\% y en los beneficios de la pandemia el 20,33\% de las opiniones de los encuestados (Tabla 4, Figura 2).

TABLA 4

Percepción de los efectos de la pandemia en la cantidad de hortalizas consumidas en los hogares de Cúcuta

\begin{tabular}{llllllll}
\hline & & & & & A5 & A6 & \\
Hortalizas & A1 $(\%)$ & A2 $(\%)$ & A3 $(\%)$ & A4 $(\%)$ & $(\%)$ & $(\%)$ & A7 $(\%)$ \\
Papa & 14,29 & 11,73 & 21,43 & 35,71 & 3,57 & 5,1 & 8,16 \\
Tomate & 7,14 & 15,31 & 21,94 & 35,71 & 4,08 & 7,14 & 8,67 \\
Cebolla & 6,63 & 15,31 & 20,92 & 36,73 & 4,08 & 6,63 & 9,69 \\
Pimentón & 7,14 & 11,73 & 20,92 & 40,82 & 5,1 & 6,12 & 8,16 \\
Ajo & 6,63 & 10,71 & 22,96 & 37,24 & 5,1 & 5,61 & 11,73 \\
Zanahoria & 6,12 & 12,76 & 19,39 & 38,78 & 7,14 & 6,63 & 9,18 \\
Promedio & 7,99 & 12,93 & 21,26 & 37,50 & 4,85 & 6,21 & 9,27 \\
\hline
\end{tabular}




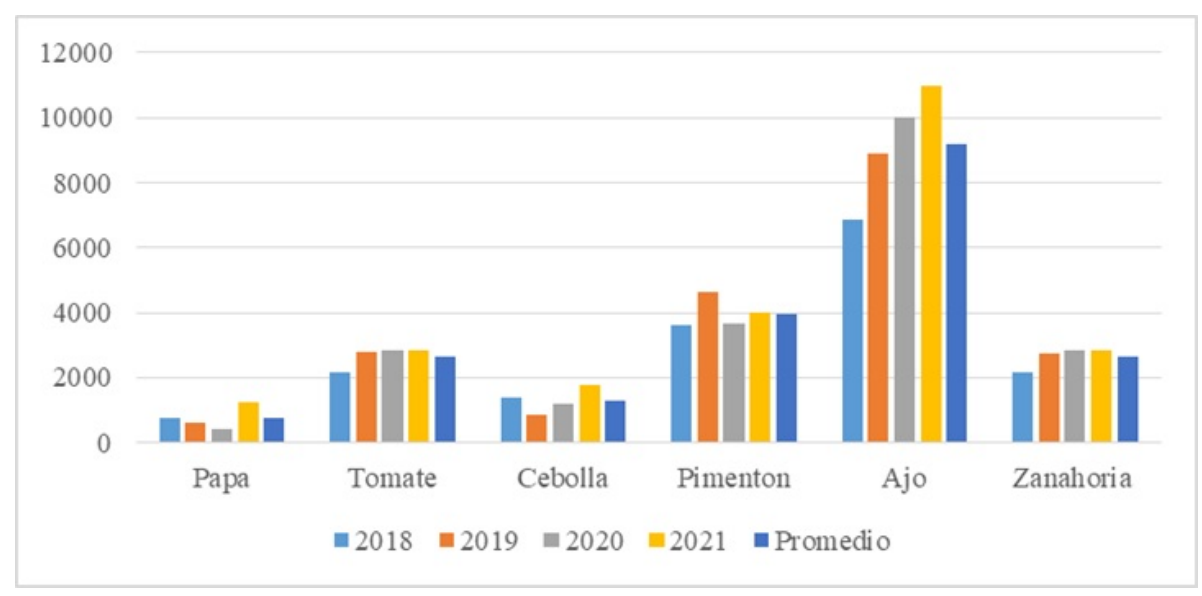

FIGURA 2 .

Percepción de los efectos de la pandemia en la cantidad de hortalizas consumidas en los hogares de Cúcuta

Estudios realizados durante la pandemia sobre la cantidad de hortalizas consumidas en hogares muestran una tendencia al incremento de frutas, legumbres y pescados (Pérez-Rodrigo et al, 2020), adaptándose a las situaciones de crisis por las restricciones en la compra de alimentos (Berges et al., 2020). Se observa una mayor valoración de los contenidos de vitaminas, minerales, fibras y fotoquímicos (Bellacomo et al., 2020), incorporándose una mayor cantidad y diversidad de hortalizas en la dieta familiar (Castagnino et al., 2020).

En Italia, Grant et al., (2021), hallaron aumentos del 28,5\% en el consumo de verduras y 22,1\% en el consumo de legumbres, mientras que, en Brasil, Carvalho et al., (2021) en un estudio realizado con adolescentes hallaron un incremento del 3,16\% en la ingesta de verduras, con respecto al consumo antes de la pandemia. No obstante, Poveda et al., (2021), en Ecuador, encontraron durante la pandemia consumos insuficientes de verduras y hortalizas del $71,7 \%$ en comedores populares.

\section{Percepción de los efectos de la pandemia del Covid-19 en la calidad de hortalizas consumidos en los hogares}

Los efectos de la pandemia del Covid-19 sobre la calidad de las hortalizas consumidas, en opinión de los encuestados, no han sido significativos. Entre las escalas de mucho a poco afectados se encuentra, que el $37,08 \%$ cree que la calidad ha sido afectada, un 34,35\% consideran que no ha sido afectada o beneficiada y el 19,99\% cree que la pandemia benefició la calidad de las hortalizas y un 8,59\% no respondió la pregunta (Tabla 5 y Figura 3)

TABLA 5.

Percepción de los efectos de la pandemia del Covid-19 en la calidad de hortalizas consumidos en los hogares

\begin{tabular}{lllllllll}
\hline & & & & & & & & Sin \\
& & & & & A5 & A6 & A7 & respuesta \\
Hortalizas & A1 $($ ) & A2 $($ ) & A3 $(\%)$ & A4 $(\%)$ & $(\%)$ & $(\%)$ & $(\%)$ & $(\%)$ \\
Papa & 11,73 & 11,73 & 16,33 & 32,14 & 5,1 & 6,63 & 8,16 & 8,16 \\
Tomate & 9,69 & 11,73 & 19,39 & 30,61 & 4,59 & 6,63 & 8,67 & 8,67 \\
Cebolla & 8,16 & 12,76 & 16,33 & 34,18 & 4,08 & 6,63 & 9,18 & 8,67 \\
Pimentón & 7,65 & 10,71 & 17,35 & 34,69 & 5,61 & 7,14 & 8,16 & 8,67 \\
Ajo & 7,65 & 11,73 & 14,8 & 37,24 & 4,08 & 6,63 & 9,18 & 8,67 \\
Zanahoria & 6,12 & 12,24 & 16,33 & 37,24 & 4,59 & 6,63 & 8,16 & 8,67 \\
Promedio & 8,5 & 11,82 & 16,76 & 34,35 & 4,68 & 6,72 & 8,59 & 8,59 \\
\hline
\end{tabular}




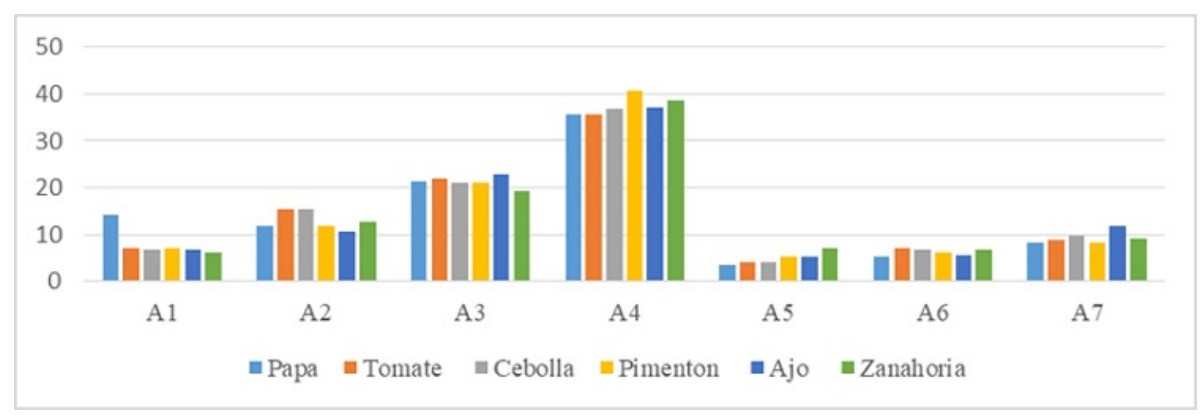

FIGURA 3

Percepción de los efectos de la pandemia del Covid-19 en la calidad de hortalizas consumidos en los hogares

Las variaciones de la calidad de las hortalizas consumidas en hogares no han sido de interés en las investigaciones reportadas en las bases de datos. Sanabria-Velásquez et al., (2020), explican que ante el cierre por confinamiento por la pandemia los comercializadores no discriminaron en la calidad de las hortalizas comprando todo lo que existía en las fincas. Asimismo, otra preocupación de los actores de la cadena productiva fue la inocuidad de los alimentos por el peligro de contagio del Covid-19 (RIMISP, 2021; Sanabria, Enciso y Maidana (2020), generando temores en la compra de productos alimenticios.

La tendencia de la calidad en las compras de hortalizas evidencia preferencias de los consumidores de obtener productos frescos adquiridos por pedidos en línea directamente de los productores (Butu et al., 2020), y compras en mercados locales o tiendas de barrio cercanas a los hogares (Pérez-Rodrigo, 2020), con énfasis en el incremento de la diversidad de hortalizas (Castagnino et al., (2020), para el fortalecimiento del sistema inmunitario de los grupos de alto riesgo ante el Covid-19 (Moreb et al., 2021).

\section{Percepción de los efectos de la pandemia del Covid-19 en los precios de las hortalizas consumidas en los hogares de la ciudad de Cúcuta}

De los 4 componentes analizados, los impactos de la pandemia del Covid-19 en los precios de las hortalizas consumidas en los hogares de la ciudad de Cúcuta, es la que evidencia el mayor efecto. El sector de las afectaciones alcanza un total del 49,59\%, siendo las opciones ha afectado mucho y medianamente el 30,54\%, es decir los encuestados consideran que los precios de las hortalizas durante la pandemia han aumentado; mientras que $26,53 \%$ opina que los precios no han afectado ni beneficiado el consumo, $12,16 \%$ cree que los precios han beneficiado y $11,73 \%$ no contestó la pregunta. La papa y el ajo son las hortalizas donde los encuestados han observado los mayores efectos de los precios y el pimentón ha sido el más estable de las 6 hortalizas analizadas (Tabla 6 y Figura 4 ) 
TABLA 6.

Percepción de los efectos de la pandemia del Covid-19

en los precios de las hortalizas consumidos en los hogares

\begin{tabular}{lllllllll}
\hline Hortalizas & A1 (\%) & A2 (\%) & A3 (\%) & A4 (\%) & $\begin{array}{l}\text { A5 } \\
(\%)\end{array}$ & $\begin{array}{l}\text { A6 } \\
(\%)\end{array}$ & $\begin{array}{l}\text { A7 } \\
(\%)\end{array}$ & $\begin{array}{l}\text { Sin } \\
\text { respuesta } \\
(\%)\end{array}$ \\
Papa & 21,94 & 15,31 & 17,35 & 22,96 & 3,06 & 4,08 & 3,57 & 11,73 \\
Tomate & 15,82 & 15,31 & 21,43 & 24,49 & 2,55 & 3,57 & 5,1 & 11,73 \\
Cebolla & 15,82 & 16,84 & 18,37 & 22,96 & 4,08 & 3,57 & 6,63 & 11,73 \\
Pimentón & 13,27 & 14,29 & 19,9 & 29,59 & 4,08 & 3,06 & 4,08 & 11,73 \\
Ajo & 17,35 & 11,22 & 17,86 & 29,08 & 2,55 & 4,59 & 5,61 & 11,73 \\
Zanahoria & 15,31 & 10,71 & 19,39 & 30,1 & 5,61 & 2,55 & 4,59 & 11,73 \\
Promedio & 16,59 & 13,95 & 19,05 & 26,53 & 3,66 & 3,57 & 4,93 & 11,73 \\
\hline
\end{tabular}

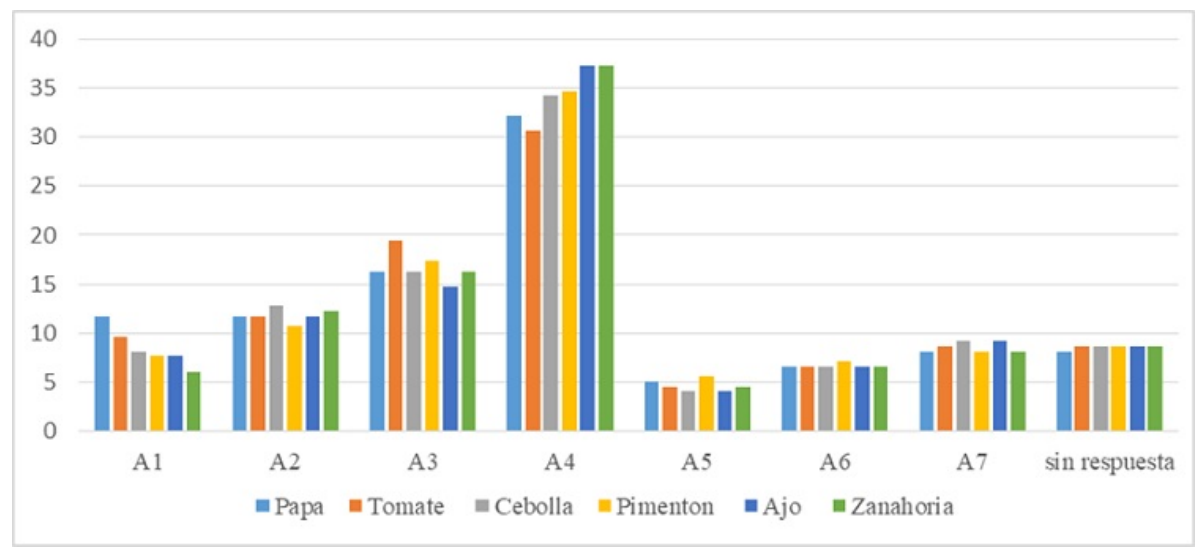

FIGURA 4

Percepción de los efectos de la pandemia del Covid-19

en los precios de las hortalizas consumidos en los hogares

Las medidas restrictivas impuestas para controlar la pandemia afectaron significativamente a la cadena agroalimentaria. En forma general la acumulación de alimentos perecederos frescos en las zonas productoras, el cierre de las cadenas de distribución y la disminución de los ingresos económicos de las familias generaron aumento en los precios de los alimentos. Algunos autores difieren en los hallazgos al establecer incrementos y disminuciones de precios durante la pandemia. Arce (2020) y Bailón-Zambrano (2020 consideran que la demanda y precios de las hortalizas cayeron por las normativas, regulaciones y protocolos de la pandemia; mientras que Ordinola y Devaux (2021), Mogues (2020), Martínez et al., (2021) consideran la presencia de presiones alcistas en los precios, por la afectación de la oferta, demanda y perdidas de los puestos de trabajos que impulsan el reemplazo del consumo de frutas, verduras y legumbres por alimentos básicos menos nutritivos.

\section{Percepción de los efectos de la pandemia del Covid-19 en la frecuencia de consumo de hortalizas en los hogares de la ciudad de Cúcuta}

La frecuencia del consumo en hogares de la ciudad de Cúcuta no ha sido afectada significativamente por la pandemia del Covid-19. El 33,08\% considera que la situación del coronavirus si ha afectado la frecuencia de comprar y consumir hortalizas, el 35,71\% opinó que no han ocurrido cambios y el 20,67\% piensa que la pandemia ha beneficiado el consumo. La gráfica 5 muestra una distribución normal de las respuestas 
centradas en la opción de no afectación ni beneficio de la pandemia sobre la frecuencia del consumo de las hortalizas en las familias encuestadas (Tabla 7 y Figura 5)

TABLA 7

Percepción de los efectos de la pandemia del Covid-19 en la frecuencia de consumo de hortalizas en los hogares de la ciudad de Cúcuta

\begin{tabular}{lllllllll}
\hline & & & & & & & & \multicolumn{3}{c}{ Sin } \\
M1 & & & & & A6 & & respuesta \\
Hortalizas & $(\%)$ & A2 $($ ) & A3 $(\%)$ & A4 $(\%)$ & A5 $(\%)$ & $(\%)$ & A7 $(\%)$ & $(\%)$ \\
Papa & 10,2 & 10,71 & 15,31 & 35,2 & 5,1 & 6,63 & 6,12 & 10,71 \\
Tomate & 7,14 & 12,76 & 12,76 & 36,73 & 8,67 & 4,59 & 6,63 & 10,71 \\
Cebolla & 6,63 & 11,22 & 15,82 & 35,71 & 5,1 & 5,61 & 9,18 & 10,71 \\
Pimenton & 8,16 & 11,73 & 13,27 & 34,69 & 7,14 & 7,14 & 7,14 & 10,71 \\
Ajo & 7,65 & 9,18 & 13,27 & 38,27 & 6,12 & 4,59 & 10,71 & 10,2 \\
Zanahoria & 7,65 & 10,71 & 14,29 & 33,67 & 11,22 & 3,57 & 8,67 & 10,2 \\
Promedio & 7,91 & 11,05 & 14,12 & 35,71 & 7,23 & 5,36 & 8,08 & 10,54 \\
\hline
\end{tabular}

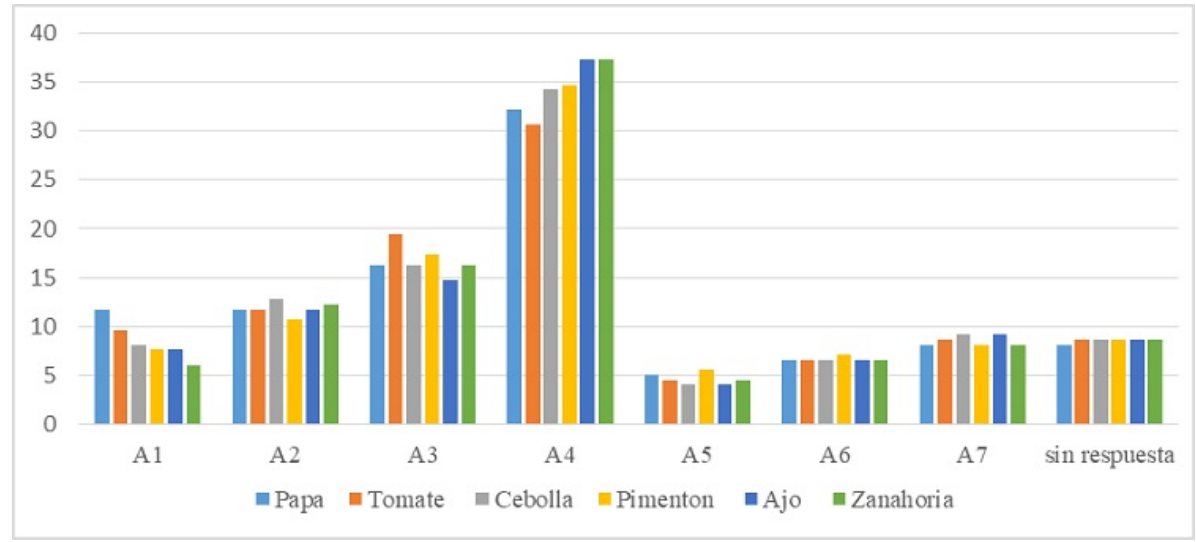

FIGURA 5.

Percepción de los efectos de la pandemia del Covid-19 en la frecuencia de consumo de hortalizas en los hogares de la ciudad de Cúcuta

Investigaciones reportadas sobre los efectos de la pandemia en la frecuencia del consumo de hortalizas evidencian aumentos, y en algunos casos, disminuciones en referencia a las directrices de la Organización Mundial de la Salud (OMS) de consumir un promedio de 5 porciones diarias de frutas, verduras y legumbres para fortalecer el sistema inmunitario (Jayawardena y Misra, 2020). Entre los hallazgos que determinaron mejoras y aumentos en las frecuencias de consumo se encuentran los estudios realizados por Berges et al., (2020), Castagnino et al., (2020),Scarmozzino y Visioli (2020) y Bilal (2020). En caso contrario, los reportes de disminución en las frecuencias de consumo de hortalizas aparecen en las investigaciones desarrolladas por Bellacomo et al., (2020) y Peng et al., (2021), mientras que Butu et al., (2020), en consumidores rumanos, observaron preferencias por compras de alta frecuencia, semanales o quincenales, durante la pandemia.

\section{Conclusiones}

En el período analizado se observan variaciones importantes en la comercialización de las hortalizas derivadas de los efectos de la pandemia del Covid-19; conflictos sociales y políticos y; sequias e inundaciones de los fenómenos del Niño y la Niña, respectivamente. Los efectos económicos de la pandemia en la oferta de alimentos se evidencian al comparar los precios de las hortalizas en los mercados mayoristas, de los años 2019 
(antes de la pandemia) y 2021 (tercer pico de la pandemia) por los incrementos significativos en los precios de la papa en un $97,90 \%$, el tomate un 2,39\%, la cebolla blanca cabezona en un 105,02\%, el ajo en un $23,79 \%$ y la zanahoria en un 2,53\%. El pimentón fue el único producto estudiado que disminuyó en un 14,49\% su precio en los mercados mayoristas. En el periodo en referencia se observan incrementos en los precios de las hortalizas por la contracción de la oferta y la demanda de alimentos derivados de las restricciones de la pandemia del Covid-19 Los efectos de la pandemia en el consumo de hortalizas en los hogares de la ciudad de Cúcuta, en la percepción de los jefes de familia, señalan que la cantidad de hortalizas consumidas han sido afectadas en un $42,48 \%$, sin efectos el $37,50 \%$ y beneficiadas por la pandemia en un 20,33\%. La cuarentena, la paralización de los procesos de comercialización y la reducción de los ingresos familiares fueron factores que influyeron en esta variable en estudio. Los efectos de la pandemia en la calidad de los productos hortícolas fueron percibidos con bajo impacto al ubicarse entre las escalas de mucho a poco afectados se encuentran el 37,08\% y el 34,35\%, respectivamente. El mayor impacto de la pandemia fue percibido en los precios de las hortalizas al ubicarse en el sector de las afectaciones el 49,59\%, siendo las opciones ha afectado mucho y medianamente el 30,54\%, es decir, los encuestados consideran que los precios de las hortalizas durante la pandemia han aumentado; mientras que $26,53 \%$ opina que los precios no han afectado ni beneficiado el consumo, $12,16 \%$ cree que los precios han beneficiado. La reducción en la oferta, las limitaciones en la comercialización y el cierre de los mercados influyeron en el incremento de los precios de las hortalizas analizadas. Los efectos del consumo en la frecuencia de las hortalizas fueron percibidos por los encuestados de bajo impacto, el 33,08\% considera que la situación del coronavirus si ha afectado la frecuencia de comprar y consumir hortalizas, el 35,71\% opinó que no habido cambios y el 20,67\% piensa que la pandemia ha beneficiado el consumo. Esto indica que a pesar de las restricciones en la producción, comercialización e ingresos económicos de las familias consultadas la frecuencia de consumo de hortalizas en la dieta diaria no fue afectada significativamente.

\section{ReFERENCIAS}

Arce, A. (2020). Sujetxs rurales: productores y productoras ante la pandemia. voces y experiencias. Estudios Rurales, v. 10, $\mathrm{n}^{\circ}$. 20. Disponible en: http://portal.amelica.org/ameli/jatsRepo/181/1811302017/index.html

AGRONET-MINAGRICULTURA (2021). Estadísticas agropecuarias. Recuperado de https:// www.agronet.gov.co/estadistica/Paginas/home.aspx

Bailón-Zambrano, M. (2020). Oferta de productos locales para efectividad comercial. Revista Científica Arbitrada de Investigación en Comunicación, Marketing y Empresa REICOMUNICAR, v. 3, nº 6, p. 2-20. DOI: https:// doi.org/10.46296/rc.v3i6.0013

Bellacomo, C.; Berriolo, M.J., Caracotche. M. ., Castagnino, A., Cendón, M., Díaz, K., Fasciglione. G., González Ferrín, M., Mairosser, A., Martinoia, G., Rogers, W., Rosini, M., Villagra, C., Yommi, A., Zazzetta, M.,L. (2020). Panorama of intensive plant production of healthy foods - "Proalim Km 0", in times of pandemic by Covid-19. Part 2 - Preferences of the production, agribusiness, commercialization and consumption of diversity of vegetables. Horticultura Argentina, v.9, n 100, pp. 357- 397.

Berges, M., Lupín, B., Rodríguez, J., Ariza, M., Mujica, G., Roldán, C., Pérez, G.,, Brillanti, C., Menéndez, L., Cutrera, G., y Agullo, A. (2020). Tendencias del consumo de alimentos durante la pandemia en el Municipio de General Pueyrredon. Póster presentado en Jornadas INVESTIGAR UNMDP-2020, Mar del Plata [ARG], 19-30 octubre 2020

Castagnino, A., Diaz, K., Rosini, M., García, F., Di Martino, B., Amendolara, P., Mariejhara, A., Bastien, E., Bosisio, M. \& Ferraris, C. (2020). Trends in regional consumption in times of Covid-19 pandemic. Horticultura Argentina v. 39, no 99, p. $43-75$.

Bilal, C. \& Senol, D. (2020). The effects of COVID-19 Pandemic Outbreak on Food Consumption Preferences and Their Causes, J Res Med Dent Sci, v. 8, nº 3, p. 169-173. 
Butu, A., Brumă, I., Tanasă, L., Rodino, S., Vasiliu, C., Sebastian Doboş, S. \& Butu, M. (2020). The Impact of COVID-19 Crisis upon the Consumer Buying Behavior of Fresh Vegetables Directly from Local Producers. Case Study: The Quarantined Area of Suceava County, Romania, International Journal of Environmental Research and Public Health, v. 17, nº. 15, 5485. DOI: https://doi.org/10.3390/ijerph17155485

Centro de Análisis y Difusión de la Economía Paraguaya (CADEP). (2020). Agricultura Familiar, ODS y Recuperación Económica post pandemia. Disponible en: http://www.soberaniaalimentaria.org.py/wpcontent/uploads/2020/10/Agricultura-Familiar-ODS-1.pdf

Carvalho, D. et al. (2021). The COVID-19 pandemic and changes in the lifestyles of Brazilian adolescents, Rev. bras. Epidemiol. 24: E210012. DOI: https://doi.org/10.1590/1980-549720210012

Departamento Administrativo Nacional de Estadística (DANE). (2019). Mercado laboral por departamentos. Recuperado de https://www.dane.gov.co/index.php/estadisticas-por-tema/mercado-laboral/mercado-laboralpor-departamentos

Diario Frontera (24-12-2019). En Norte de Santander hay 183.215 venezolanos a octubre. Recuperado de https:// www.laopinion.com.co/frontera/en-norte-de-santander-hay-183215-venezolanos-octubre-189265

Diario La Opinión (11-10-2019). Cúcuta sigue líder en informalidad laboral con 73,3\%. Recuperado de https:// www.laopinion.com.co/economia/cucuta-sigue-lider-en-informalidad-laboral-con-733-185241\#OP

Diario La Opinión (26-11-2019). Mercado laboral, el gran reto para la competitividad de Norte de Santander. Recuperado de https://www.laopinion.com.co/economia/mercado-laboral-el-gran-reto-para-lacompetitividad-de-norte-de-santander-187712

Grant, F., Scalvedi, M., Scognamiglio, U., Turrini, A. \& Rossi, L. (2021). Eating Habits during the COVID-19 Lockdown in Italy: The Nutritional and Lifestyle Side Effects of the Pandemic, Nutrients v. 13, nº.7, 2279. DOI: https://doi.org/10.3390/nu13072279

Gobernación del departamento Norte de Santander (2016). Plan de Desarrollo para Norte de Santander 2016-2019. "Un Norte Productivo Para Todos". Recuperado de http://www.sednortedesantander.gov.co/sitio/images/ documentos/informesdelsector/PDD\%20NDS\%202016-2019.pdf

González, C. (2011). Comparisons of LSMS-ISA data collection and dissemination efforts in Central America. Journal of development and Agricultural Economics, v, 3, n 8, pp. 353-361.

Gutiérrez, M., Suarez, M. y Villalba, A. (2021). Producir y comercializar en pandemia: estrategias emergentes de los agricultores familiares en Santiago del Estero durante 2020. Trabajo y sociedad, v. 22, no . 37, p. 13-30. Disponible en: https://www.redalyc.org/journal/3873/387368391002/html/

Hernández, R., Fernández, C. y Baptista, P. (2014). Metodología de la Investigación, Editorial MC Graw Hill, Quinta Edición, México

Jayawardena, R. \& Misra, A. (2020). Balanced diet is a major casualty in COVID-19. Diabetes \& metabolic syndrome, v. 4, no 5, p. 1085-1086. DOI: https://doi.org/10.1016/j.dsx.2020.07.001

Martínez, A., Aguilera, C., Hernández, J., Ruiz, J. y Mireles, A. (2021). Percepción e impacto del COVID 19 en el sector agroalimentario del estado de Guanajuato, Jóvenes en la ciencia, v. 10, p. 1-9. Disponible en: https:// www.jovenesenlaciencia.ugto.mx/index.php/jovenesenlaciencia/article/view/3374/2874

Ministerio de Agricultura y Desarrollo Rural (MINAGRICULTURA). (2019). Evaluaciones estadísticas agropecuarias (EVA). Recuperado de https://www.upra.gov.co/web/guest/evaluaciones-agropecuariasmunicipales-eva

Mogues, T. (2020). Los mercados de alimentos durante la COVID-19, IMF, Fiscal Affairs, Disponible: file://D:/ Documents/OTROS\%20ARCHIVOS/sp-special-series-on-covid-19-food-markets-during-covid-19.PDF

Moreb, N., Ahmed, A., Swarna, J. \& Amit J. (2021). Fruits and Vegetables in the Management of Underlying Conditions for COVID-19 High-Risk Groups, Foods v. 10, nº. 2, 389. DOI: https://doi.org/10.3390/ foods10020389

Núñez, J. y Carvajal, J. (2014). Los perfiles tecnológicos del comercio rural nortesantandereano: un análisis del potencial competitivo de los principales rubros agropecuarios, informe final de Proyecto de investigación, Universidad de Santander, Cúcuta. 
Núñez, J., Carvajal, J., Carrero, D., Mendoza, O. (2018). Indicadores del impacto del cambio climático en la agricultura familiar andina colombiana, Revista Iberoamericana de Bioeconomía y Cambio Climático, v. 4, nº 7, p. 824-833. DOI: https://doi.org/10.5377/ribcc.v4i7.6309

Ordinola, M. y Devaux, A. (2021). Desafíos y oportunidades para el sector papa en la zona andina en el contexto de la COVID-19. Revista Latinoamericana de la Papa, v. 25, nº 1, p. 102-124. DOI: https://doi.org/10.37066/ ralap.v25i1.422

Organización de las Naciones Unidas para la Agricultura y la Alimentación (FAO). (2020). Nueva enfermedad por coronavirus (COVID-19). Recuperado de http://www.fao.org/2019-ncov/es/

Organización Mundial del Comercio (OMC). (2020). COVID-19 y comercio mundial. Recuperado de https:// www.wto.org/spanish/tratop_s/covid19_s/covid19_s.htm

Peng, J., Liang, L., Xiaofen, X., Changzheng, Y., Hui, C., Bing, G., Junmin, Z. \& Shujuan, Y. (2021). Changes in dietary patterns among youths in China during COVID-19 epidemic: The COVID-19 impact on lifestyle change survey (COINLICS). Appetite, v. 158, 105015. DOI: https://doi.org/10.1016/j.appet.2020.105015

Pérez-Rodrigo, C. et al. (2020). Cambios en los hábitos alimentarios durante el periodo de confinamiento por la pandemia COVID-19 en España, Rev Esp Nutr Comunitaria, v. 26, no 2), p.101-111. DOI:10.14642/ RENC.2020.26.2.5213

Poveda, C., Peré, G., Jouvín, J., Celi, M. y Yaguachi, R (2021). Prácticas alimentarias y estilos de vida en la población de Guayaquil durante la pandemia por Covid-19. Nutrición Clínica Y Dietética Hospitalaria, V. 41, No 3. DOI: https://doi.org/10.12873/413poveda

Programa de Naciones Unidas para el Desarrollo (PNUD). (2019). Norte de Santander: retos y desafíos por el Desarrollo Sostenible. Recuperado de https://www.undp.org/content/dam/colombia/docs/Gobernabilidad/ Publicacionesproyectos/UNDP_Co_GOB_Publicaciones_FICHA\%20NORTE\%20DE\%20SANT\%20\%20RETOS\%20Y\%20DESAF\%C3\%8DOS\%20PARA\%20EL\%20DESARROLLO.pdf

Programa Especial para la Seguridad Alimentaria (PESA). (2011). Seguridad Alimentaria y Nutricional. Conceptos Básicos. Centroamérica, Proyecto Food Facility Honduras. Recuperado de http://www.fao.org/3/a-at772s.pdf

Programa Mundial de Alimentos (PMA). (2008). La vulnerabilidad alimentaria de hogares desplazados y no desplazados: un estudio de caso en ocho departamentos de Colombia. https://www.acnur.org/fileadmin/ Documentos/Publicaciones/2008/6821.pdf

Programa Mundial de Alimentos (PMA). (2020). La pandemia por COVID-19. Recuperado de https://es.wfp.org/ emergencias/pandemia-covid-19

Centro Latinoamericano para el Desarrollo Rural (RIMISP). (2021). Evolución del costo de los alimentos ante el COVID-19, Análisis de Coyuntura covid-19 en América Latina, Análisis n¹5. Disponible en: https:// opendocs.ids.ac.uk/opendocs/handle/20.500.12413/16650

Sanabria-Velázquez, A., Enciso-Maldonado, G. y Maidana, M. (2020). Medidas para minimizar los efectos del SARs-CoV-2 en la horticultura paraguaya. Actualidad del Campo Agropecuario, nota técnica. Disponible: https://www.researchgate.net/ publication/341273915_Medidas_para_minimizar_los_efectos_del_SARs-

CoV-2_en_la_horticultura_paraguaya

Scarmozzino, F. \& Visioli, F. (2020). Covid-19 and the Subsequent Lockdown Modified Dietary Habits of Almost Half the Population in an Italian Sample, Foods, v. 9, nº. 5, 675. DOI: https://doi.org/10.3390/foods 9050675

Tikitakas (2020). Cuarentena total en Colombia: qué es, cuándo empieza y cuándo acaba. Recuperado de https:// colombia.as.com/colombia/2020/05/20/tikitakas/1589996857_168861.html

Universidad Nacional de Rosario (2021). Informe semestral Canasta Básica Alimentaria. Ciudad de Rosario. Segundo semestre de 2020. Disponible en: http://rephip.unr.edu.ar/bitstream/handle/2133/19564/Informe \%20Semestral\%20CBA\%202021-01.pdf?sequence=3\&isAllowed=y 